\title{
Intradermal Therapy (Mesotherapy) for the Treatment of Acute Pain in Carpal Tunnel Syndrome: A Preliminary Study
}

\author{
Emergency Department, *Neurology Department, Central General Hospital, Bolzano-Bozen, Italy
}

Giorgio Conforti, Loredana Capone*, and Stefano Corra

\section{Background:}

The carpal tunnel syndrome (CTS) is the most common cause of severe hand pain. In this study we treated acute pain in CTS patients by means of local intradermal injections of anti-inflammatory drugs (mesotherapy).

\section{Methods:}

In twenty-five patients (forty-five hands), CTS diagnosis was confirmed by clinical and neurophysiological examination prior to mesotherapy. A mixture containing lidocaine $10 \mathrm{mg}$, ketoprophen lysine-acetylsalycilate $80 \mathrm{mg}$, xantinol nicotinate $100 \mathrm{mg}$, cyanocobalamine 1,000 mcg plus injectable water was used. Sites of injection were three parallel lines above the transverse carpal ligament and two v-shaped lines, one at the base of the thenar eminence, and the other at the base of the hypothenar eminence.

\section{Results:}

The day after the treatment, all but four patients reported a significant reduction in pain and paresthesias. After 12 months, 17 patients had a complete pain relief, eight patients reported recurrence of pain and sensory symptoms and four out of them underwent surgical treatment.

\section{Conclusions:}

With the obvious limits of a small-size open-label study, our results suggest that mesotherapy can temporary relieve pain and paresthesias in most CTS patients and in some cases its effect seems to be long-lasting. Further controlled studies are needed to confirm our preliminary findings and to compare mesotherapy to conventional approaches for the treatment of CTS. (Korean J Pain 2014; 27: 49-53)

\section{Key Words:}

carpal tunnel syndrome, intradermal therapy, mesotherapy. 


\section{INTRODUCTION}

Carpal tunnel syndrome (CTS) is the most common entrapment neuropathy in the upper extremity and has an estimate prevalence of $3-16 \%[1,2]$. The pathophysiological mechanism of CTS is the increased pressure within the carpal tunnel, that results from increases in the volume of canal's contents, particularly of the flexor tenosynovium [3]. Chronic nerve compression produces focal demyelination and in more severe cases there is an axonal degeneration of the nerve fibers [4]. Pain, paresthesias and sensory impairment in the median nerve distribution territory are the most frequent symptoms in CTS. Patients often wake up at night because of pain and numb hand sensation. Non-operative management is usually the first line of treatment and includes generally oral non-steroidal anti-inflammatory drugs, injections of medications, rehabilitation techniques and wrist splinting [5-9]. In more severe cases, the surgical division of the transverse carpal ligament, performed in the classic open technique or endoscopically, is the main treatment of syndrome $[10,11]$.

Mesotherapy is the practice of using microinjections of medication into mesoderm or middle layer of skin to treat a specific area of body [12]. Local bleeding, swelling, infections, allergy are the most common adverse effects of mesotherapy [13,14]. A growing body of literature supports the efficacy of mesotherapy, particularly in the treatment of musculoskeletal pain [15-20]. In patients with chronic back pain due to musculoskeletal conditions, pain is triggered both by inflammation and by dysfunction of the nerve pathways (neuropathic pain) [21]. On the base of the promising results of mesotherapy in patients with chronic back pain $[15,16]$, we decided to evaluate the efficacy of this approach for the treatment of pain and sensory symptoms in CTS, where neuropathic pain represents the main component of the symptom.

\section{MATERIALS AND METHODS}

All the patients that presented to the Emergency Department between January 2008 and December 2009 with clinical symptoms of CTS, where considered for this study. Patients with known or suspected polyneropathy, diabetes, any medical condition causing chronic pain (including chronic cervical or lumbar pain), psychiatric diseases, use of anticoagulants, chronic renal failure, coagulation dis- order and liver cirrosis were not enrolled. Twenty-five patients (21 females, 45 hands, mean age 56, range: 23-84), were selected. Eight patients were on anti-hypertensive therapy (two on beta-blocker, three on sartanic and three on a combination of ACE-inhibitor and diuretic) and their treatment was obviously not interrupted during the study. Mesotherapy technique was explained to the patients and written consent was obtained. All patients were clinically evaluated using the Simovic and Weinberg Quantitative Clinical Scale (SWQCS) for CTS (Table 1) [22]. This scale investigates clinical signs (sensory loss in median nerve distribution territory, weakness and atrophy of thenar muscles, occurrence of Phalen sign and Tinel sign), subjective symptoms (pain in hand, forearm and arm, paresthesias, weakness and clumsiness in the hand), worsening and improving conditions. All patients performed a standard neurophysiological examination to confirm the diagnosis [23]. Electrodiagnostic measurements were performed with a Medelec Synergy (Oxford Instruments Inc., UK) electromyography device. Nerve conduction was investigated using surface electrodes. Median sensory distal latencies were recorded orthodromically at the wrist after stimulation of the first four fingers and palm. Ulnar sensory distal latencies were recorded at the wrist by stimulation of the fourth and fifth finger. Median motor conduction studies were performed using a supramaximal percutaneous stimulation at the wrist and elbow with a recording electrode placed on the thenar muscles. Ulnar motor conduction studies were performed by nerve stimulation at the wrist and over the cubital sulcus with a recording electrode placed on the hypothenar muscles. Compound

Table 1. Simovic and Weinberg Quantitative Clinical Scale for CTS [22]

1 dull aching pain in the hand or arm: 1 point

2 paresthesias or numbness in the hand: 1 point

3 weakness or clumsiness in the hand: 1 point

4 above symptoms in isolated median nerve distribution: 4 points

5 symptoms provoked by sleep: 2 points

6 symptoms provoked by sustained hand position or repetitive hand action: 2 points

9 Tinel or Phalen sign: 5 points

10 sensory loss isolated to the median nerve distribution: 5 points 11 weakness or atrophy of the thenar muscles: 5 points

Total score; $1-6$ points: unlikely CTS, $7-8$ points: possible CTS, 9-30 points: probable CTS. 
Table 2. Padua's Neurophysiologic Severity Scale for CTS [20]

\begin{tabular}{cllll}
\hline Score & Severity & $\mathrm{mSDL} / \mathrm{uSDL}$ & $\mathrm{mSDL}$ & $\mathrm{mMDL}$ \\
0 & Negative & Normal & Normal & Normal \\
1 & Minimal & Pathological & Normal & Normal \\
2 & Mild & Pathological & Pathological & Normal \\
3 & Moderate & Pathological & Pathological & Pathological \\
4 & Severe & Not evaluable & Absent & Pathological \\
5 & Extreme & Not evaluable & Absent & Absent \\
\hline
\end{tabular}

$\mathrm{mSDL} / \mathrm{uSDL}$ : difference between median sensory distal latency and ulnar sensory distal latency, mSDL: median sensory distal latency, uSDL: ulnar sensory distal latency, mMDL: median motor distal latency.

muscle action potentials and nerve sensory action potentials, amplitudes and distal latencies were measured. Criteria for the diagnosis of CTS were a median motor distal latency greater than $4.2 \mathrm{~ms}$, a median sensory distal latency greater than $3.4 \mathrm{~ms}$ at fingers and greater than $2.0 \mathrm{~ms}$ at palm [24]. A difference between median and ulnar sensory latencies at fourth finger greater than $0.4 \mathrm{~ms}$ is considered a sensitive diagnostic criterion for mild carpal tunnel syndrome [25,26]. Ulnar motor distal latencies greater than $4.0 \mathrm{~ms}$ and sensory distal latencies greater than $3.4 \mathrm{~ms}$ were considered pathological. The Padua's Neurophysiologic Severity Scale (PNSS) for CTS was used to assess severity of the median fibers damage (Table 2) [27].

The clinical and neurophysiological examinations were perfomed on both hands in each patient, but only hands affected by CTS were treated. Mesotherapy was performed using disposable devices each containing three $4 \mathrm{~mm}$ long 27 G needles (Mesoram ${ }^{\circledR}$, Ri.Mos. srl, Italy). Each hand received a single treatment session. For each treatment session a pharmacological mixture containing the following drugs was prepared: lidocaine $10 \mathrm{mg}$, to keep the solution at a constant $\mathrm{pH}$ and to minimize pain at site of injection, ketoprofen lysine-acetylsalycilate $80 \mathrm{mg}$ as pain reliever, xantinol nicotinate $100 \mathrm{mg}$ as vasodilating agent to increase tissue perfusion, cyanocobalamine $1,000 \mathrm{mcg}$ as neurotrophic agent, betamethasone disodium phosphate 4 $\mathrm{mg}$ to reduce the inflammatory response plus injectable water enough to reach $10 \mathrm{ml}$ of solution. Sites of injection were three parallel lines above the transverse carpal ligament, each line with six points injections (double injection with a triple needle), and two $\mathrm{v}$-shaped lines, one at the base of the thenar eminence, and the other at the base of the hypothenar eminence, both with six points injections (Fig. 1). Each injection contained $0.6 \mathrm{ml}$ (0.2 ml per point

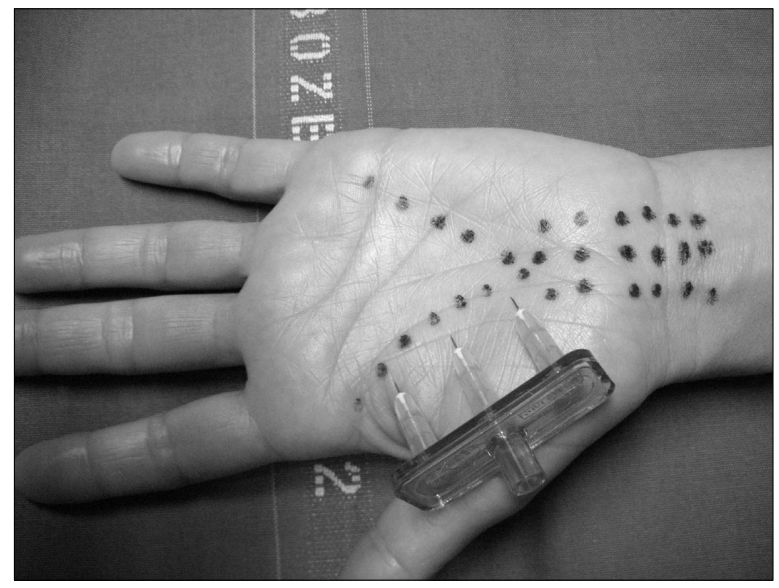

Fig. 1. Sites of injections.

of injection) and the whole treatment included $6 \mathrm{ml}$. of solution. Injections were performed with the needle positioned at 90 degrees on the skin surface. These sites were chosen to allow the drugs to adequately spread both in the region of the median nerve, and to the palm of the hand.

The day after the treatment all patients underwent clinical examination of the hand and were asked about symptoms resolution. After 12 months all patients were contacted by phone and asked about recurrence or resolution of symptoms.

\section{RESULTS}

Clinical and neurophysiological evaluation confirmed CTS in all patients. The mean duration of symptoms was 7.8 months (range: 10 days -3 years). The mean score at the SWQCS was 18.7/30 (range: 7-27). The mean score at the PNSS was $3 / 5$ (range: 1-5). The right hand was more affected in 12 patients, the left hand in eight patients 
and both hands were equally affected in five patients. The day after mesotherapy, all but four patients reported a significant reduction of sensory symptoms and the quality of sleep immediately improved. Four patients with the highest SWQCS scores, who also complained of clumsiness and weakness of the hand, did not report improvement of the symptoms. The main side-effect of the treatment complained by all patients was short-lasting pain at the injection sites. One patient had transient facial flushing.

After one year 17 patients had a complete pain relief and reported an improvement of sensory impairment. These patients had a mean SWQCS score of 16. Eight patients with a mean SWQCS score of 20 suffered from recurrent pain and paresthesias. Four of them underwent surgical treatment, two with a complete relief of symptoms. No difference at the PNSS scores was found among patients who benefited from mesotherapy and those who did not.

\section{DISCUSSION}

To our knowledge this is the first study that evaluates the effectiveness of anti-inflammatory drugs administered via mesotherapy in patients with CTS. This is a small-size open-label study with its obvious limits. Nevertheless, our results show that mesotherapy could provide a valuable contribution to the treatment of pain and sensory symptoms in CTS, particularly among those patients with less severe clinical signs (SWQCS score of 16 or less), and its effectiveness seems to be long lasting. Previous studies demonstrated that injection of drugs via mesotherapy into the dermis slowly achieves higher local concentrations in the underlying tissuses, as compared to intramuscular or subcutaneous administration [17,28]. Therefore, mesotherapy allows the use of reduced dosage of anti-inflammatory drugs, minimizing their adverse events.

It is still under debate whether local therapy or surgical approach represent the best treatment for CTS. Steroids injection into the carpal tunnel was recently compared with surgery in a controlled trial [29]. The results of conservative treatment seems to be worse as compared to decompressive surgery, particularly in the function recovery of the median nerve [30]. Some authors, however, emphasize the good results of steroid injection in patients with less severe symptoms [31]. Injection of low dosage of anti-inflammatory drugs and steroids via mesotherapy could represent an alternative choice to conventional approaches for the treatment of acute pain and sensory symptoms in CTS patients. However, further controlled studies are required. The choice of the drugs used in this study was based empirically both on previously published studies [14], and on the bases of the known mechanism of action of the drugs, when administered according to conventional medicine. Further studies are also needed to find out whether other drugs or other dosages would have better effectiveness.

\section{REFERENCES}

1. de Krom MC, Knipschild PG, Kester AD, Thijs CT, Boekkooi PF, Spaans F. Carpal tunnel syndrome: prevalence in the general population. J Clin Epidemiol 1992; 45: 373-6.

2. Atroshi I, Gummesson C, Johnsson R, Ornstein E, Ranstam J, Rosén I. Prevalence of carpal tunnel syndrome in a general population. JAMA 1999; 282: 153-8.

3. Gelberman RH, Hergenroeder PT, Hargens AR, Lundborg GN, Akeson WH. The carpal tunnel syndrome. A study of carpal canal pressures. J Bone Joint Surg Am 1981; 63: 380-3.

4. You H, Simmons Z, Freivalds A, Kothari MJ, Naidu SH. Relationships between clinical symptom severity scales and nerve conduction measures in carpal tunnel syndrome. Muscle Nerve 1999; 22: 497-501.

5. Boyd KU, Gan BS, Ross DC, Richards RS, Roth JH, MacDermid JC. Outcomes in carpal tunnel syndrome: symptom severity, conservative management and progression to surgery. Clin Invest Med 2005; 28: 254-60.

6. Falkenburg SA. Choosing hand splints to aid carpal tunnel syndrome recovery. Occup Health Saf 1987; 56: 60, 63-4.

7. Muller M, Tsui D, Schnurr R, Biddulph-Deisroth L, Hard J, MacDermid JC. Effectiveness of hand therapy interventions in primary management of carpal tunnel syndrome: a systematic review. J Hand Ther 2004; 17: 210-28.

8. Shi Q, MacDermid JC. Is surgical intervention more effective than non-surgical treatment for carpal tunnel syndrome? A systematic review. J Orthop Surg Res 2011; 6: 17.

9. Chang MH, Chiang HT, Lee SS, Ger LP, Lo YK. Oral drug of choice in carpal tunnel syndrome. Neurology 1998; 51 : 390-3.

10. Semple JC, Cargill AO. Carpal-tunnel syndrome. Results of surgical decompression. Lancet 1969; 1: 918-9.

11. Shum C, Parisien M, Strauch RJ, Rosenwasser MP. The role of flexor tenosynovectomy in the operative treatment of carpal tunnel syndrome. J Bone Joint Surg Am 2002; 84-A: 221-5.

12. Rohrich RJ. Mesotherapy: what is it? Does it work? Plast Reconstr Surg 2005; 115: 1425.

13. Bessis D, Guilhou JJ, Guillot B. Localized urticaria pigmentosa 
triggered by mesotherapy. Dermatology 2004; 209: 343-4.

14. Brandão C, Fernandes N, Mesquita N, Dinis-Ribeiro M, Silva $\mathrm{R}$, Lomba Viana $\mathrm{H}$, et al. Abdominal haematoma--a mesotherapy complication. Acta Derm Venereol 2005; 85: 446.

15. Di Cesare A, Giombini A, Di Cesare M, Ripani M, Vulpiani MC, Saraceni VM. Comparison between the effects of trigger point mesotherapy versus acupuncture points mesotherapy in the treatment of chronic low back pain: a short term randomized controlled trial. Complement Ther Med 2011; 19: 19-26.

16. Costantino C, Marangio E, Coruzzi G. Mesotherapy versus systemic therapy in the treatment of acute low back pain: a randomized trial. Evid Based Complement Alternat Med 2011; 2011: 317183

17. Cacchio A, De Blasis E, Desiati P, Spacca G, Santilli V, De Paulis F. Effectiveness of treatment of calcific tendinitis of the shoulder by disodium EDTA. Arthritis Rheum 2009; 61: 84-91.

18. Mammucari M, Gatti A, Maggiori S, Sabato AF. Role of mesotherapy in musculoskeletal pain: opinions from the italian society of mesotherapy. Evid Based Complement Alternat Med 2012; 2012: 436959.

19. Mammucari M, Gatti A, Maggiori S, Bartoletti CA, Sabato AF. Mesotherapy, definition, rationale and clinical role: a consensus report from the Italian Society of Mesotherapy. Eur Rev Med Pharmacol Sci 2011; 15: 682-94.

20. Navarte DA, Rosset-Llobet J. Safety of subcutaneous microinjections (mesotherapy) in musicians. Med Probl Perform Art 2011; 26: 79-83.

21. Freynhagen R, Baron R, Tölle T, Stemmler E, Gockel U, Stevens M, et al. Screening of neuropathic pain components in patients with chronic back pain associated with nerve root compression: a prospective observational pilot study (MPORT). Curr Med Res Opin 2006; 22: 529-37.

22. Simovic D, Weinberg DH, Allam G, Hayes MT. A quantitative clinical scale for the carpal tunnel syndrome. 50th Annual
Meeting of the American Academy of Neurology (AAN). Minneapolis, 25 April-2 May 1998, Poster 05.119. Neurology 1998; 50: 5119.

23. Jablecki CK, Andary MT, Floeter MK, Miller RG, Quartly CA, Vennix MJ, et al. Practice parameter: electrodiagnostic studies in carpal tunnel syndrome. Report of the American Association of Electrodiagnostic Medicine, American Academy of Neurology, and the American Academy of Physical Medicine and Rehabilitation. Neurology 2002; 58: 1589-92.

24. Kimura J. Electrodiagnosis in diseases of nerve and muscle: principles and practice. 2nd ed. Philadelphia (PA), Oxford University Press. 1989, pp 501-11.

25. Cioni R, Passero S, Paradiso C, Giannini F, Battistini N, Rushworth G. Diagnostic specificity of sensory and motor nerve conduction variables in early detection of carpal tunnel syndrome. J Neurol 1989; 236: 208-13.

26. Uncini A, Lange DJ, Solomon M, Soliven B, Meer J, Lovelace RE. Ring finger testing in carpal tunnel syndrome: a comparative study of diagnostic utility. Muscle Nerve 1989; 12 : 735-41.

27. Padua L, Lo Monaco M, Valente EM, Tonali PA. A useful electrophysiologic parameter for diagnosis of carpal tunnel syndrome. Muscle Nerve 1996; 19: 48-53.

28. Hsu CC, Kuo HC, Hsu CT, Gu Q. Abdominal mesotherapy injection extended the absorption of follicle-stimulating hormone. Fertil Steril 2011; 95: 2134-6, 2136.e1.

29. Andreu JL, Ly-Pen D. A randomized controlled trial of surgery vs steroid injection for carpal tunnel syndrome. Neurology 2006; 66: 955-6.

30. Hui AC, Wong S, Leung CH, Tong P, Mok V, Poon D, et al. A randomized controlled trial of surgery vs steroid injection for carpal tunnel syndrome. Neurology 2005; 64: 2074-8.

31. Hoffman DE. Treatment of carpal tunnel syndrome: is there a role for local corticosteroid injection? Neurology 2006; 66: 459-60. 